Research Article

\title{
Ameliorative Effects of Gallic Acid on Cisplatin-Induced Nephrotoxicity in Rat Variations of Biochemistry, Histopathology, and Gene Expression
}

\author{
Zahra Eslamifar $\mathbb{D}^{1},{ }^{1}$ Abbas Moridnia ${ }^{(D)}, 2$ Susan Sabbagh $\left(\mathbb{D},{ }^{3}\right.$ Reza Ghaffaripour $\mathbb{D}^{\mathrm{D}},{ }^{1}$ \\ Leila Jafaripour $\left(\mathbb{D},{ }^{3}\right.$ and Mahin Behzadifard $\mathbb{1}^{1}$ \\ ${ }^{1}$ Department of Medical Laboratory Sciences, School of Paramedical Sciences, Dezful University of Medical Sciences, Dezful, Iran \\ ${ }^{2}$ Department of Immunology, School of Medicine, Dezful University of Medical Sciences, Dezful, Iran \\ ${ }^{3}$ Department of Anatomy, Faculty of Medicine, Dezful University of Medical Sciences, Dezful, Iran \\ Correspondence should be addressed to Zahra Eslamifar; eslamifar.z@gmail.com
}

Received 26 July 2021; Revised 18 September 2021; Accepted 21 September 2021; Published 26 October 2021

Academic Editor: Fatma M. El-Demerdash

Copyright (c) 2021 Zahra Eslamifar et al. This is an open access article distributed under the Creative Commons Attribution License, which permits unrestricted use, distribution, and reproduction in any medium, provided the original work is properly cited.

\begin{abstract}
Background. Cisplatin is a powerful chemotherapeutic drug mainly used in the treatment of solid tumors. Aggregation of the drug in renal proximal tubule cells causes nephrotoxicity and renal failure. Investigations showed nephrotoxicity as Cisplatin's doselimiting side effect. One of the Cisplatin toxicity mechanisms is generation of reactive oxygen species, which leads to oxidative stress and renal damage. The purpose of this study was evaluation of the modulating effects of Gallic acid on Cisplatin-induced variations including Caspase-3 and Clusterin expression and histopathological and biochemical parameters in adult male Wistar rats. Method. Rats were kept under standard condition of temperature, light, and humidity. The animals were divided into 4 groups: GpI: control group (received distilled water for 10 days); GpII: Gallic acid (alone) (50 mg/kg bw, once a day for 10 days); GpIII: Cisplatin (alone), single dose $(6 \mathrm{mg} / \mathrm{kg}$ bw, I.P. on 5 th day of study); GpIV: Gallic acid ( $50 \mathrm{mg} / \mathrm{kg}$ bw, once a day for 10 days) and also injected with single dose of Cisplatin $(6 \mathrm{mg} / \mathrm{kg}$ bw, I.P., on 5 th day of study). After 10 days, all rats were anaesthetized and plasma collected to estimate urea, creatinine, and uric acid. The right kidneys were removed for the study of gene expression and biochemical parameters. The left kidneys were used for histopathological studies. Results. The Cisplatin-induced nephrotoxicity was evident from the elevated levels of creatinine, urea, uric acid, and renal tissue MDA and also decreased levels of SOD, CAT, GPX, and GSH in renal tissue. Administration of Gallic acid significantly modulated nephrotoxicity markers, gene expression variations, and histopathological damage. Conclusion. Outcomes of the present investigation suggest that Gallic acid provides protection against CP-induced nephrotoxicity, but for application in people, further studies are needed.
\end{abstract}

\section{Introduction}

Cisplatin (cis-diamminedichloroplatinum-II) is a powerful chemotherapeutic drug predominantly used in the treatment of solid tumors [1].

The kidney in addition to playing its role as eliminator of endogenous and exogenous waste materials, including drugs, stores some of these substances in the proximal tubular section. In the treatment with Cisplatin (CP), renal tissue accumulates this drug to a higher level than other tissues and organs.
Cisplatin concentration in the epithelial cells of renal proximal tubules was detected to be about 5 times higher than serum [2]. Aggregation of the drug leads to strong toxicity in renal proximal tubule cells and finally causes tissue destruction, low perfusion, and renal failure [3, 4]. Low renal perfusion indicates nephrotoxicity and necrosis of the terminal portion of the proximal tubule and finally determines renal tissue fate [5].

Nearly $25-35 \%$ of subjects following a single dose of Cisplatin develop evidence of renal dysfunction [6]. Preclinical 
investigations showed nephrotoxicity and renal dysfunction as one of Cisplatin's dose-limiting side effects [5].

Acute renal failure is a considerable motivating force of mortality and morbidity development among Cisplatin chemotherapy recipient subjects. Nephrotoxicity is distinguished by tubular dilation, tubular cell vacuolization, loss of microvilli, and condensation of nuclear chromatin. Tubular destruction can be the loss of only the margin of the epithelial cell brush to tubular necrosis in severe renal damage. Diminished glomerular filtration and enhancement of blood urea nitrogen and plasma creatinine represent Cisplatininduced renal failure [6-9].

Multifactorial mechanisms are involved in Cisplatininduced nephrotoxicity including the production of free radicals such as superoxide and hydroxyl radicals, mitochondrial dysfunction, and increased activity of calcium independent nitric oxide synthase and ultimately apoptosis. One of the mechanisms and processes that have been detected to play Cisplatin toxicity is its impact on DNA synthesis and repairs, which causes cell cycle inhibition $[9,10]$.

Mitochondrial irregularity is a key event in Cisplatininduced acute renal failure. Cisplatin collection in the mitochondria causes the generation of reactive oxygen species leading to oxidative stress resulting in nephrotoxicity and renal damage $[11,12]$.

Investigations have also presented that Cisplatin induces the tumor-suppressor protein p53. This affects apoptosis via the receptor-tumor necrosis factor interaction, and Caspases that cause mitochondrial disorder also affect the calcium signaling by stress on the endoplasmic reticulum $[9,13,14]$.

Despite the side effects of Cisplatin, it is the preferred treatment in chemotherapy due to its high efficacy and low cost [15].

There are several anti-inflammatory and antioxidant factors that have ameliorative effects on the potential side effects of Cisplatin [16-18].

3,4,5-Trihydroxybenzoic acid called Gallic acid (GA) is an antioxidant product, present in some natural ingredients including green tea, red wine, pineapples, strawberries, lemons, bananas, sumac, gallnuts, tea leaves, witch hazel, apple peels, and oak bark [19-21].

Gallic acid, as a powerful chelating factor, protects tissues against oxidative stress due to antioxidant and antiinflammatory effects [22-24]. This valuable compound does not only maintain the integrity of cell membrane but also increases the regenerative capacity of the kidney and liver [25]. Furthermore, Gallic acid and its derivations have anticancer properties due to antioxidant and anti-inflammatory features [26].

Clusterin gene expression is upregulated in response to numerous types of renal toxicity and diseases [27]. Wang et al. showed that Clusterin gene expression was highly induced in rats after treatment with Cisplatin [28].

Reactive oxygen species show a significant role in apoptosis by inducing the Caspase activity. Among the Caspase family, Caspase-3 specially is a main apoptotic effector leading to cytoskeletal decomposition, nuclear destruction, and other changes associated with apoptosis [29, 30].
Mechanisms related to inducing the Caspase-3 activity are all known to be involved in Cisplatin-induced tubular apoptosis. Caspase inhibition markedly reduces nephron injury in Cisplatin therapy [31].

The recognition of natural antioxidants and their possible function on side effects of chemotherapeutic drugs provides a valuable plan in the treatment of patients.

The main purpose of our study was to estimate the modulating effects of Gallic acid on Cisplatin-induced variations on Caspase-3 and Clusterin expression and nephrotoxicity in adult male Wistar rats.

\section{Materials and Methods}

Cisplatin and Gallic acid were purchased from Sigma Chemical Co (St Louis, MO, USA). The Gallic acid was dissolved in distilled water just before the experiments. Other materials used were of analytical grade.

2.1. Animals. Healthy adult male Wistar rats (12-14 weeks old; weighing $200 \pm 20 \mathrm{~g}$ ) were purchased from the Animal House of Ahvaz Jundishapur University of Medical Sciences, Ahvaz, Iran. The rats were housed in clean polypropylene cages with free access to standard rat chow and water under controlled conditions of humidity $(65 \% \pm 5)$, temperature $\left(25 \pm 2^{\circ} \mathrm{C}\right)$, and air ventilation with a $12 \mathrm{~h}$ light/dark cycle. The maintenance protocol of animals was approved by the Institutional Animal Ethics Committee (Registration number IR.DUMS.REC.1398.008; Proposal No. 1532: dated 06.02.2019), Dezful University of Medical Sciences, Dezful, Iran.

2.2. Experimental Design. 32 male Wistar rats were divided into 4 groups including experimental groups and control group (8 rats in each group).

Group I received distilled water for 10 days as the control group (cnt group). Group II (gal group) was administered by gavage with Gallic acid alone $(50 \mathrm{mg} / \mathrm{kg}$ bw, once a day for 10 days) starting from day 1 and continuing daily for 10 days.

Group III (cis group) was injected intraperitoneally with a single dose of Cisplatin alone $(6 \mathrm{mg} / \mathrm{kg}$ bw, injection on 5 th day).

Group IV (cis-gal group) was administered by gavage with Gallic acid ( $50 \mathrm{mg} / \mathrm{kg}$ bw, once a day for 10 days) and also injected with a single dose of Cisplatin $(6 \mathrm{mg} / \mathrm{kg} \mathrm{bw}$, I.P., on 5 th day of study).

All rats were anaesthetized with diethyl ether on the 10th day of investigation, and then, blood samples from the heart were collected in heparinized vials, and plasma was used to estimate of biochemical parameters (B. urea, P. creatinine, and P. uric acid using commercially available diagnostic kits).

The right kidneys were removed and frozen in $-70^{\circ} \mathrm{C}$ until homogenization and the study of gene expression and biochemical parameters. The left kidneys were placed in $10 \%$ formalin for histopathological studies.

2.3. Investigations of Biochemical Parameters. 10 times the phosphate-buffered saline (PBS) was added to the 
homogenized kidney. It was centrifugated at $4^{\circ} \mathrm{C}$ with $12000 \mathrm{rpm}$ for 15 minutes. The supernatant was used to measure malondialdehyde (MDA), glutathione (GSH), glutathione peroxidase (GPX), superoxide dismutase (SOD), and catalase (CAT). In the present study, the contents of malondialdehyde (MDA) of renal tissue were evaluated according to the thiobarbituric acid (TBA) method, similar to the method described by Buege and Aust [32]. Absorption was read at $532 \mathrm{~nm}$ by spectrophotometer (Hitachi's U-2000 Double-Beam UV/Vis Spectrophotometer). Renal levels of GSH were determined in accordance with the Ellman method [33]. GPX activity was measured by the Rotruck et al. method. In brief, tert-butyl hydroperoxide and hydrogen peroxide $\left(\mathrm{H}_{2} \mathrm{O}_{2}\right)$ were mixed with the kidney sample, and then, its absorption was read by the ELISA reader at $420 \mathrm{~nm}[34,35]$. SOD activity was measured by the Minami et al. method [36]. For the CAT reaction, $0.7 \mathrm{ml}$ of potassium phosphate buffer ( $\mathrm{pH}: 7.0$ ), $0.1 \mathrm{ml}$ of hydrogen peroxide $\left(\mathrm{H}_{2} \mathrm{O}_{2}\right)$, and $100 \mu \mathrm{l}$ of a homogenized renal sample were mixed, and its absorption at $240 \mathrm{~nm}$ was read by a spectrophotometer (Hitachi's U-2000 Double-Beam UV/Vis Spectrophotometer). [37].

2.4. Histopathological Investigations. The rat's left renal tissue was removed, washed with ice-cold physiological saline solution, and fixed in $10 \%$ neutral buffered formalin solution for $24 \mathrm{~h}$. Routine processing and paraffin embedding were done, and the tissue was sectioned at $4-5 \mu \mathrm{m}$ thickness by a rotary microtome. Sections were stained with hematoxylin-eosin (H\&E) dyes and observed in a magnification of 100x under a light microscope (Olympus BX 52, Tokyo, Japan) [38].

For each kidney, composed of slides of control and experimental groups (at least 50 samples), the glomerulus diameter and thickness of proximal convoluted tubule (PCT) were measured by using an image analysis software; the Motic Images Plus 2.0 and the average of measured data were calculated.

Additionally, five stained sections of each renal tissue were assessed for these criteria: vacuolization of proximal cell, congestion of red blood cells, leukocyte infiltration, eosinophilic casts necrosis of PCT cells, and hemorrhage. Then, the percentage of acute renal tubular necrosis was classified and scored by the semiquantitative method, such as 0 point: normal; 1 point: $<10 \%$; 2 points: $10-25 \%$; 3 points:26-75\%; and 4 points: $>75 \%$. Finally, for each measure, the average percentage was determined [39].

2.5. RNA Extraction, cDNA Synthesis, and Real-Time PCR. The total RNA was extracted from rat kidney tissue using BioFACT $^{\text {тм }}$ Total RNA Prep Kit (Ver. 2.0, South Korea), according to the manufacturer's instruction. In the first step, liquid nitrogen was added to the samples and tissues were crushed separately. The samples were transferred to RNase-free tubes and stored on ice. Then, $1 \mathrm{ml}$ of tissue lysis buffer was added to the tubes that were homogenated by an ultrasonic sonicator. Finally, the RNA purity was checked with a NanoDrop ND-1000 UV-Vis Spectrophotometer (Thermo Fisher Scientific, Waltham, MA, USA). In addition, complementary DNAs (cDNAs) were synthesized by the BioFact $^{\mathrm{TM}}$ RT Series Kit (BioFact, South Korea) according to the manufacturer's instructions. The real-time qPCR was done for CASP3 and Clusterin gene expression using the SYBR Green Master Mix Kit (BioFact, South Korea). The GAPDH gene was used for internal control and normalization. The primers were as follows: Clusterin F: $5^{\prime}$ TACA ACGAGCTGCTTCATTC 3', R: 5' AGAATGGGTTGTCA CTGTGG 3'; CASP3 F: 5' CTGGACTGTGGCATTGAGA C 3', R: 5' TCCAGGAATAGTAACCAGGTG 3'; and GAPDH F: 5' CTCATGCGACTTCAACAGC 3', R: 5' CGTCTACATTGTCATACCAGG 3'. The ABI StepOnePlus (Applied Bio systems, Foster City, CA, USA) instrument and $\Delta \Delta \mathrm{Ct}$ method were used for data analysis of the qPCR experiment.

2.6. Statistical Analysis. Data were analyzed by the ShapiroWilk normality test for normality. Parameters that showed normal distribution were used as a one-way analysis of variance (ANOVA). In case of significance between groups, Tukey's multiple-comparison posttest was used, and the data are expressed as mean \pm standard deviation (SD). Nevertheless, data that did not show a normal distribution used nonparametric tests such as Kruskal-Wallis tests, and the data were expressed as median (min-max). Statistical analysis was performed by GraphPad Prism 8.0. A statistical probability of $P$ value $<0.05$ was considered statistically significant.

\section{Results}

3.1. Ameliorative Effects of Gallic Acid on Plasma Kidney Parameters. The effects of Gallic acid (50 mg/kg bw, orally) on Cisplatin- (6 mg/kg bw, IP) induced nephrotoxicity were evaluated by the study of plasma biochemical parameters.

The nephrotoxicity effect of Cisplatin was evident from the elevated levels of plasma renal markers including P. creatinine, B. urea, and P. uric acid observed in the cis group (Figure 1). It was demonstrated that glomerular filtration was damaged. Administration of Gallic acid prior to and following Cisplatin treatment significantly decreased nephrotoxicity (cis-gal group IV, compared to cis group III) (Figure 1, $P<0.0001$ ).

3.2. Ameliorative Effects of Gallic Acid on Renal Tissue Lipid Peroxidation. Lipid peroxidation was investigated in terms of thiobarbituric acid reactive substances (TBARS), expressed as the malondialdehyde (MDA) level. In this study, MDA were remarkably increased in the kidney tissue of the cis group compared to the cnt group $(P<0.0001)$. The elevated MDA level was ameliorated by treatment with Gallic acid $(P<0.0001)$ (cis group III compared to cis-gal group IV) (Figure 2). The administration of Gallic acid alone did not increase the level of MDA compared to the control rats. Indeed, the MDA concentrations were similar in the cnt group and gal group $(P<0.05)$. Figure 2 shows the results in the 4 groups. 


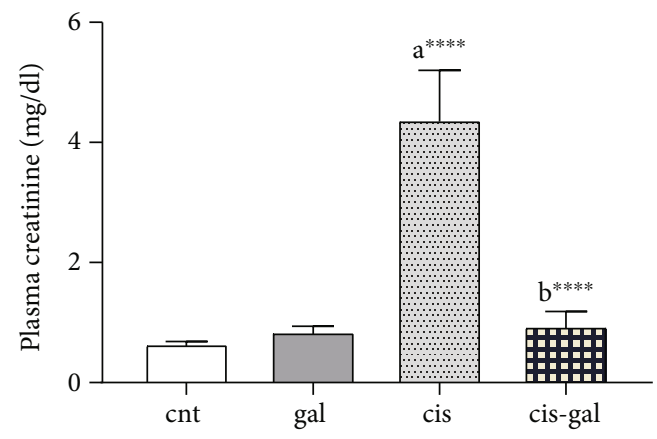

a: vs. cnt b: vs. cis

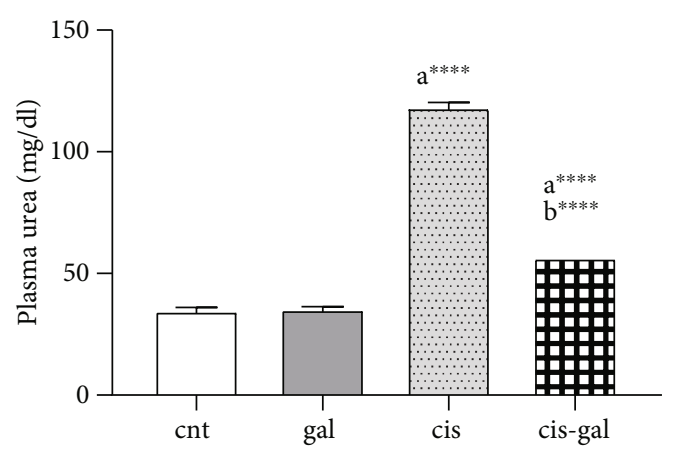

a: vs. cnt

b: vs. cis

(a)

(b)

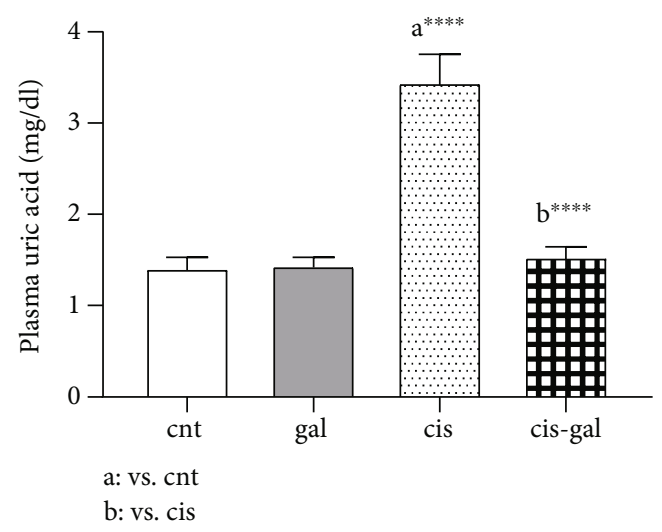

(c)

Figure 1: Effect of Gallic acid on Cisplatin-induced nephrotoxicity as measured by (a) plasma creatinine, (b) plasma urea, and (c) plasma uric acid. Nephrotoxicity in rats was induced by a single dose of Cisplatin (6 mg/kg bw, IP) and Gallic acid (50 mg/kg bw, gavage) administered 5 days prior to CP treatment and continued till the end of the experiment ( 5 days). ${ }^{* * * *} P<0.0001$; values are expressed as mean $\pm \mathrm{SD}(n=6)$. The results show that Gallic acid attenuated Cisplatin-induced renal toxicity.

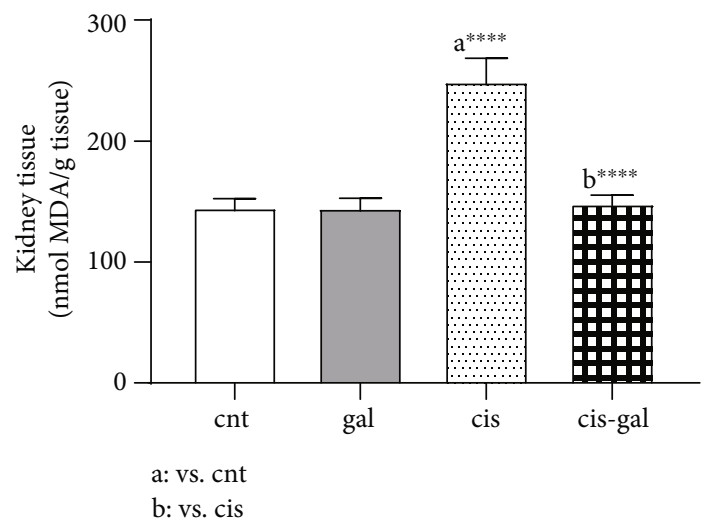

Figure 2: Effect of Gallic acid on Cisplatin-induced lipid peroxidation (kidney tissue MDA). Rats were injected with Cisplatin and Gallic acid as described in Figure 1. ${ }^{* * * *} P<0.0001$; values are expressed as mean $\pm \mathrm{SD}(n=6)$. The results show that Gallic acid treatment ameliorated the Cisplatin-induced increase in lipid peroxidation.

3.3. Effects of Gallic Acid Treatment on Renal Tissue Antioxidant Parameters. For evaluating the role of Gallic acid in attenuation of Cisplatin-induced oxidative stress, the effect of Cisplatin and Gallic acid alone and in combination was investigated on enzymatic and nonenzymatic antioxidant defense parameters.

The effect of treatment with Gallic acid on Cisplatininduced alterations in the levels of kidney tissue GSH and enzymatic antioxidant parameters including SOD, CAT, and GPX are shown in Figure 3.

Compared to the control, the rats injected with Cisplatin were shown to be remarkably reduced in renal tissue GSH $(P<0.0001)$. Treatment of animals with Gallic acid ameliorated the Cisplatin-mediated decrease in GSH (cis group vs. cis-gal group, $P<0.01$ ).

Gallic acid did not induce a statistically significant increase in kidney tissue glutathione compared to control $(P<0.05)$.

The levels of antioxidant enzyme activity were also reduced significantly in Cisplatin-treated animals compared to control (SOD $P<0.0001$, CAT $P<0.0001$, and glutathione peroxidase $P<0.001)$ and also increased in rats treated with Gallic acid plus Cisplatin compared to the cis group (SOD $P<0.01$, CAT $P<0.0001$, and glutathione peroxidase $P<0.01)$.

Therefore, it was observed that oral administration of Gallic acid prior to and following Cisplatin treatment 


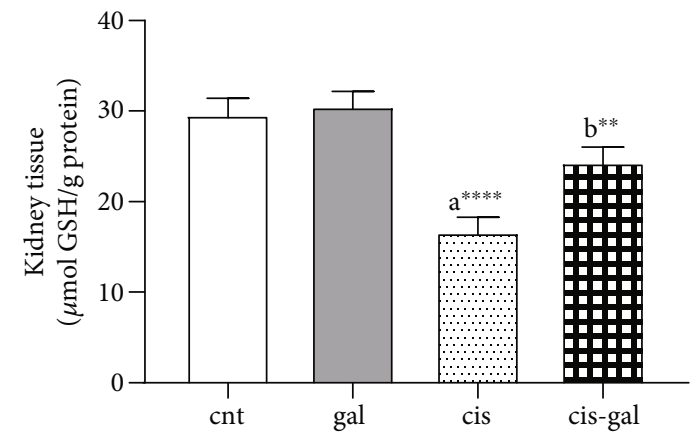

a: vs. cnt

b: vs. cis

(a)

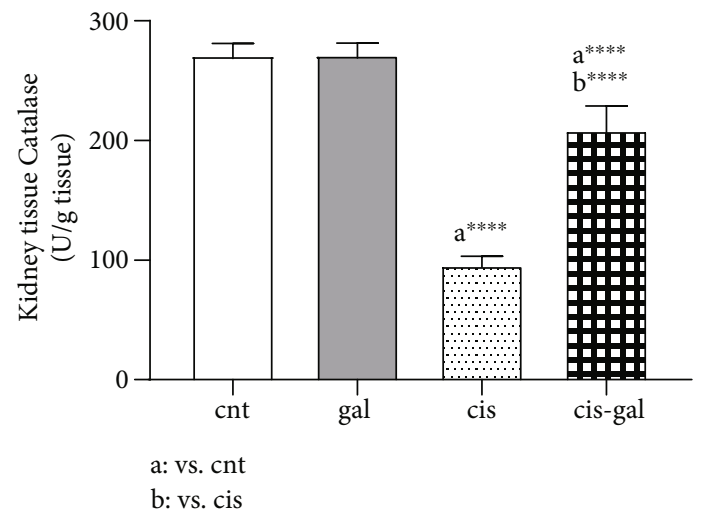

(c)

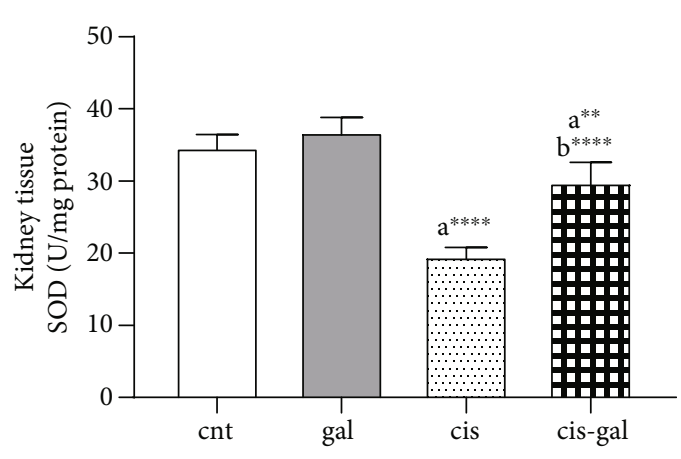

a: vs. cnt

b: vs. cis

(b)

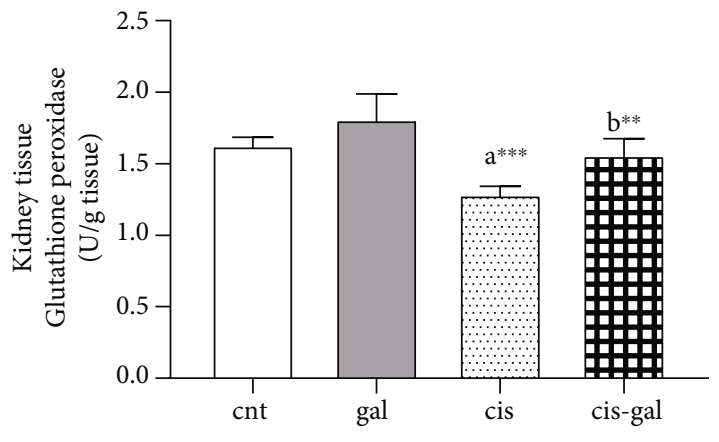

a: vs. cnt

b: vs. cis

(d)

FIGURE 3: Effect of Gallic acid on Cisplatin-induced decrease in (a) GSH, (b) SOD, (c) CAT, and (d) GPX in kidney tissue. Rats were treated with Cisplatin and Gallic acid as described in Figure $1 .{ }^{* *} P<0.01,{ }^{* * *} P<0.001$, and ${ }^{* * * *} P<0.0001$; values are expressed as mean \pm SD $(n=6)$. The results show that Gallic acid treatment ameliorated the Cisplatin-induced decrease in SOD, catalase, GSH, and glutathione peroxidase.

considerably ameliorated Cisplatin-induced decrease in the activities of the antioxidant enzyme.

\subsection{Effects of Gallic Acid Treatment on Renal Tissue} Histopathological Changes. The histopathological changes of administration of Cisplatin and Gallic acid in kidney tissues are shown in Figure 4.

One-way ANOVA did not show a significant difference between the groups in terms of the diameter of glomeruli $(F(3,17)=2.73, P=0.076)$ (Table 1$)$. One-way ANOVA showed a significant difference between the groups in terms of the thickness of proximal convoluted tubules $(F(3,15)=6.81, P=0.004)$. In this study, thickness of proximal convoluted tubules was significantly reduced in the Cisplatin group compared to the control group $(P=0.002)$. Nevertheless, no significant difference was observed in the Cisplatin group vs. the Cisplatin-Gallic group $(P=0.077)$ and the control group vs. the Gallic group $(P=0.124)$ (Table 1).

A Kruskal-Wallis test showed a significant difference among the groups in terms of the cell necrosis in the kidney $(P=0.002)$. Cell necrosis was significantly increased in the Cisplatin group compared to the Control group $(P=0.006)$.
Cell necrosis was significantly decreased in the CisplatinGallic group compared to the Cisplatin group $(P=0.001)$ (Table 1).

In the present study, the Kruskal-Wallis test showed a significant difference among the groups in the eosinophilic casts in the kidney $(P<0.0001)$. In the study, eosinophilic casts have a significant difference in the control group compared with the Cisplatin group $(P=0.006)$ and the Cisplatin group compared with Cisplatin-Gallic group $(P=0.001)$ (Table 1).

In this study, analyzing the vacuolization using a Kruskal-Wallis test revealed a significant difference among the groups $(P=0.003)$. Vacuolization was significantly increased in the Cisplatin group compared to the Control group $(P=0.006)$. Vacuolization was significantly decreased in the Cisplatin-Gallic group compared to the Cisplatin group $(P=0.008)$ (Table 1$)$.

In addition, a Kruskal-Wallis test showed a significant difference in the congestion $(P=0.014)$, hemorrhage $(P<0.0001)$, and leukocyte infiltration $(P<0.0001)$. However, no significant difference was observed in the control group vs. Cisplatin group and the Cisplatin group vs. Cisplatin-Gallic group (Table 1). 


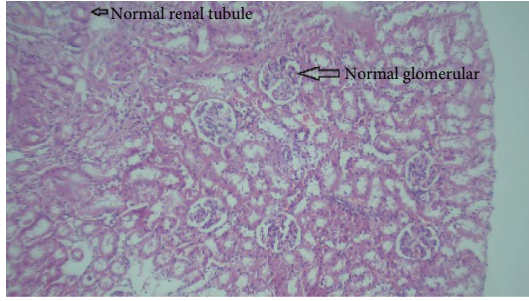

(a)

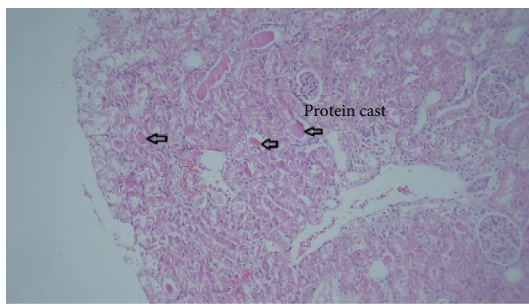

(c)

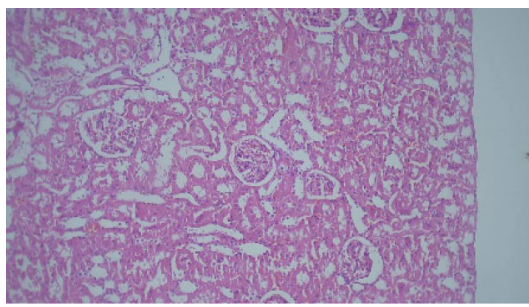

(e)

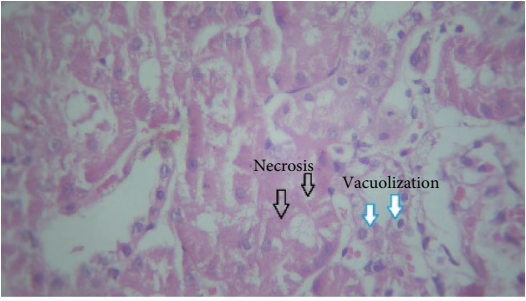

(b)

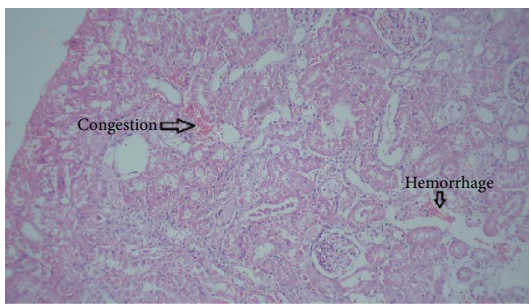

(d)

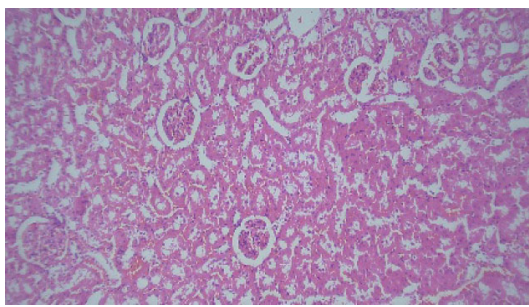

(f)

Figure 4: H\&E staining of renal tissues, magnification $\times 100$. (a) Control group, light microscopy examination of renal tissues in control group showed normal structure of glomerulus, renal cortex, and interstitium with no evidence of acute cell necrosis. Photomicrograph of a renal section taken from Gallic acid-supplemented rats had almost normal structure of glomerulus, renal cortex, and interstitium without acute necrosis $(\mathrm{b}-\mathrm{d})$. But photomicrograph of a renal section taken from cisplatin-treated rats showing marked vacuolization, necrosis of tubular cells, protein cast, and congestion of vessels (e). Photomicrograph of a renal section taken from Gallic acidsupplemented cisplatin-treated rats showing that treatment with Gallic acid remarkably attenuated renal tissue injury (f).

3.5. Effects of Gallic Acid on Expression Level of CASP3 and Clusterin Genes in Rat Kidney Tissue. The expression levels of CASP3 and Clusterin genes were assessed by RT-qPCR in the control (cnt), Gallic acid (gal), Cisplatin (cis), and Cisplatin+Gallic acid (cis-gal) groups after ten days of treatment. The cis-gal group showed significant downregulation in CASP3 mRNA expression in rat kidney tissue compared to the cis group alone $(P<0.05)$ (Figure 5$)$. The expression level of the Clusterin gene was not detectable in all groups.

\section{Discussion}

Cisplatin is still an effective and practical anticancer agent. However, in $30 \%$ of patients, it is clinically associated with acute kidney injury $[9,40]$.

This antitumor drug inhibits the mitochondrial respiratory complex in renal tubular cells, resulting in generation of reactive oxygen species (ROS) and finally tissue and organ damage [41].

The ROS generation and oxidative stress in renal tissue have resulted in lipid peroxidation and changes in enzymatic and nonenzymatic antioxidant system and the gene expression $[9,42,43]$.

Irregularities in the structure and function of renal tissue are believed to be associated with inflammation and oxidative stress production. Inflammation was caused through chemokines such as tumor necrosis factor [9]. Some studies report that apoptosis is involved in CP-induced nephrotoxicity [44-46].

Some antioxidant compounds prevented CP-induced toxicity such as melatonin [47], selenium [48], vitamin E and vitamin C $[43,49,50]$, some plants [51], modulators of nitric oxide, agents interfering with Cisplatin metabolic pathways, and antiapoptotic and cytoprotective agents [16, 52-54].

Several phenolic acid compounds, such as aqueous extract of Dendrobium nobile Lindl, protocatechuic acid, and ferulic acid, have shown conservation against nephrotoxicity and hepatotoxicity induced by Cisplatin [55-57].

Gallic acid as an antioxidant is a phenolic acid compound that can scavenge peroxyl radicals and oxidative damage in tissues [58, 59]. Numerous investigations have shown that Gallic acid has considerable antioxidant activity [60] and exhibits anti-inflammatory feature in oxidative conditions that can cause tissue damage $[61,62]$.

Gallic acid prevents methotrexate-induced nephrotoxicity [23] and sodium arsenite-induced toxicity in the rat's liver and kidney [63].

In renal tissue, Cisplatin is converted into more reactive molecules. Therefore, the production of ROS, oxidative 


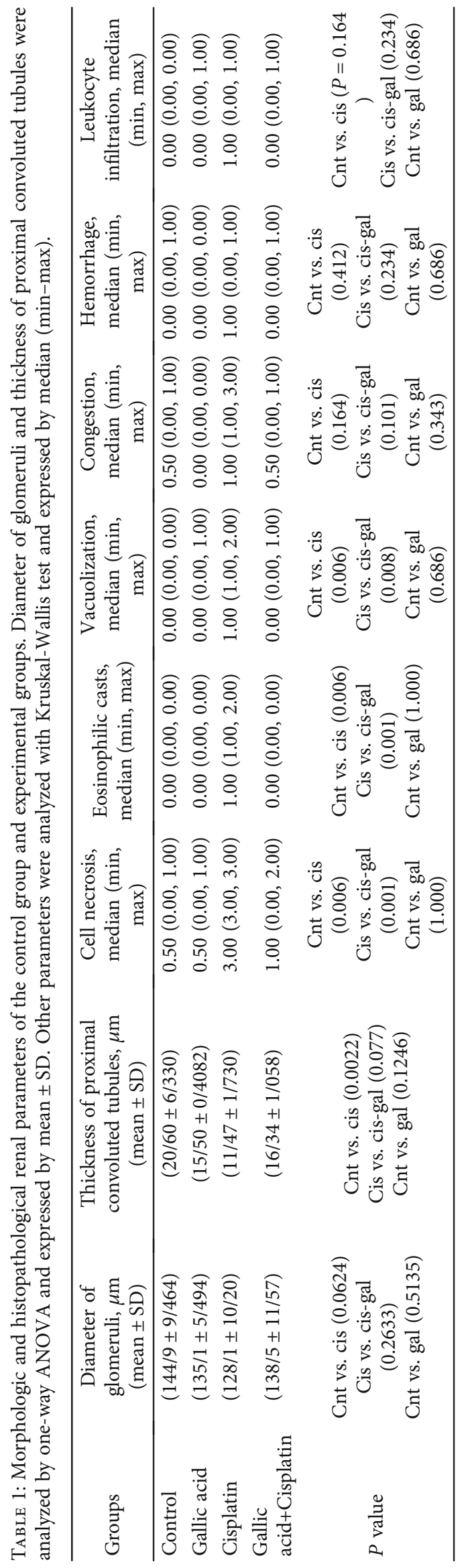




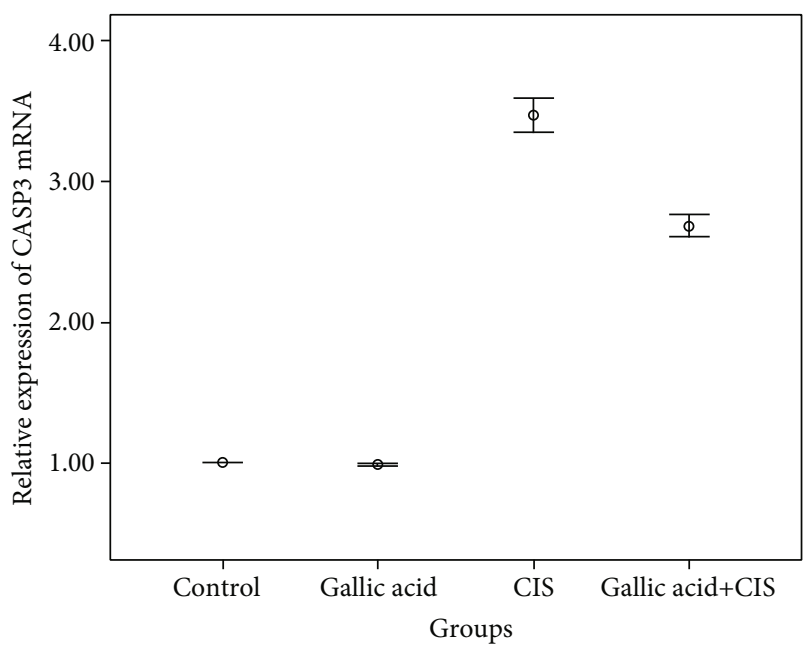

FIGURE 5: Effect of Gallic acid on Cisplatin-induced increase in expression of CASP3 in kidney tissue. Rats were treated with Cisplatin and Gallic acid as described in Figure 1. The results show that Gallic acid treatment improved the Cisplatin-induced increase in gene expression.

stress, accumulation of MDA, and depletion of antioxidant enzyme activity occur. GSH is a significant ROS scavenger and is required to maintain cell safety. CAT and GPX decompose $\mathrm{H}_{2} \mathrm{O}_{2}$, and the dismutation of the superoxide anion is done by SOD. SOD, CAT, and GPX are antioxidant enzymes that are necessary to improve kidney function [64].

Disorder in the kidney function by Cisplatin is demonstrated via the attenuation of renal tissue enzyme function such as SOD, CAT, and GPX and the increase of B. urea, $\mathrm{P}$. uric acid, and P. creatinine and also MDA generation [30, 65-67].

In this study, nephrotoxicity was induced by injecting a single I.P. dose of Cisplatin ( $6 \mathrm{mg} / \mathrm{kg}$ bw) [68]. Renal damage was confirmed by variation of the gene expression of Caspase- 3 and histological changes along with increased nephrotoxicity markers including P. creatinine, P. uric acid, B. urea, and lipid peroxidation marker (MDA).

We indicated that the use of Gallic acid significantly improved renal function with an ameliorating effect on biochemistry and histopathological marker and the expression of Caspase-3 in Cisplatin-induced nephrotoxicity.

This further confirms the antioxidative feature of Gallic acid in Cisplatin nephrotoxicity.

The anti-inflammatory and antioxidant feature of Gallic acid may play a key role of modulatory effect in Cisplatininduced toxicity $[62,69,70]$.

Apoptosis due to reperfusion ischemia and treatment with melatonin by prevention of Caspase- 3 activity has also been reported [71].

Also, it was reported that C-phycocyanin significantly inhibited the doxorubicin-induced ROS generation and apoptosis by decreasing Caspase-3 activity [72].

The present study showed that the expression of Caspase- 3 was downregulated in the cis-gal group that was confirmed by qRT-PCR. Therefore, the protective effect of orally prescribed Gallic acid, as a natural antioxidant having free radical scavenging effects, was demonstrated by the prevention of Caspase-3 activity.

These preclinical findings present Gallic acid to light as a nephroprotective agent against Cisplatin in male Wistar rats.

\section{Conclusion}

The common method for cancer treatment is chemotherapy with Cisplatin, but this drug has side effects such as nephrotoxicity.

Our study showed the modulatory effect of Gallic acid as an antioxidant in rat kidney tissue. Accordingly, Gallic acid can be an excellent treatment scope in reducing the oxidative stress and finally severity of Cisplatin-induced nephrotoxicity.

These studies are important since Cisplatin can affect the reabsorptive functions of the renal tissue leading to acute kidney injury.

Although the outcomes of the present investigation suggest that Gallic acid provides protection against CP-induced renal toxicity by the prevention of oxidative stress, alternative possible mechanisms cannot be ruled out.

In conclusion, Gallic acid markedly reduces Cisplatininduced nephrotoxicity, but further studies are necessary to exclude the fact that Gallic acid may affect the therapeutic efficacy of Cisplatin in people. In the absence of such evidence, despite the marked renal protection, definitive evaluation of the potential therapeutic importance of Gallic acid in people as an adjuvant in chemotherapy cannot be practical.

\section{Data Availability}

Upon reasonable request, the data supporting the results of this article will be made available by the corresponding responsible author.

\section{Conflicts of Interest}

The authors declare that there is no conflict of interest.

\section{Authors' Contributions}

Zahra Eslamifar, Abbas Moridnia, Susan Sabbagh, and Reza Ghaffaripour designed this project. Zahra Eslamifar, Abbas Moridnia, Susan Sabbagh, Reza Ghaffaripour, Leila Jafaripour, and Mahin Behzadifard collected data and analyzed the data. Zahra Eslamifar, Abbas Moridnia, Susan Sabbagh, and Leila Jafaripour wrote the manuscript, modified the English version, and edited the final draft.

\section{Acknowledgments}

The authors greatly appreciate the assistance of the staff of the research laboratory in conducting this research and Miss Aida Asbahi, for her assistance in collecting the data. Financial support for this project was provided by Dezful University of Medical Sciences, Iran. 


\section{References}

[1] A. Brown, S. Kumar, and P. B. Tchounwou, "Cisplatin-based chemotherapy of human cancers," Journal of cancer science \& therapy, vol. 11, no. 4, 2019.

[2] M. Kuhlmann, G. Burkhardt, and H. Köhler, "Insights into potential cellular mechanisms of cisplatin nephrotoxicity and their clinical application," Nephrology, dialysis, transplantation: official publication of the European Dialysis and Transplant Association-European Renal Association., vol. 12, no. 12, pp. 2478-2480, 1997.

[3] J. L. Digby, T. Vanichapol, A. Przepiorski, A. J. Davidson, and V. Sander, "Evaluation of cisplatin-induced injury in human kidney organoids," American Journal of Physiology-Renal Physiology., vol. 318, no. 4, pp. F971-F978, 2020.

[4] A. Naqshbandi, M. W. Khan, and S. Rizwan, "Studies on the protective effect of dietary fish oil on cisplatin induced nephrotoxicity in rats," Food and Chemical Toxicology., vol. 50, no. 2, pp. 265-273, 2012.

[5] I. Arany and R. L. Safirstein, Cisplatin nephrotoxicity, Seminars in nephrology, Elsevier, 2003.

[6] X. Han, J. Yue, and R. W. Chesney, "Functional TauT protects against acute kidney injury," Journal of the American Society of Nephrology., vol. 20, no. 6, pp. 1323-1332, 2009.

[7] Z. Duan, G. Cai, J. Li, and X. Chen, "Cisplatin-induced renal toxicity in elderly people," Therapeutic advances in medical oncology., vol. 12, article 175883592092343, 2020.

[8] A. Naqshbandi, S. Rizwan, and F. Khan, "Dietary supplementation of flaxseed oil ameliorates the effect of cisplatin on rat kidney," Journal of Functional Foods., vol. 5, no. 1, pp. 316326, 2013.

[9] S. Manohar and N. Leung, "Cisplatin nephrotoxicity: a review of the literature," Journal of nephrology., vol. 31, no. 1, pp. 1525, 2018.

[10] V. Volarevic, B. Djokovic, M. G. Jankovic et al., "Molecular mechanisms of cisplatin-induced nephrotoxicity: a balance on the knife edge between renoprotection and tumor toxicity," Journal of Biomedical Science, vol. 26, no. 1, pp. 1-14, 2019.

[11] H. Pan, J. Chen, K. Shen et al., "Mitochondrial modulation by epigallocatechin 3-gallate ameliorates cisplatin induced renal injury through decreasing oxidative/nitrative stress, inflammation and NF-kB in mice," PLoS One, vol. 10, no. 4, article e0124775, 2015.

[12] M. R. Noh, K. Y. Kim, S. J. Han, J. I. Kim, H.-Y. Kim, and K. M. Park, "Methionine sulfoxide reductase A deficiency exacerbates cisplatin-induced nephrotoxicity via increased mitochondrial damage and renal cell death," Antioxidants \& redox signaling., vol. 27, no. 11, pp. 727-741, 2017.

[13] M. I. Yousef and H. M. Hussien, "Cisplatin-induced renal toxicity via tumor necrosis factor- $\alpha$, interleukin 6 , tumor suppressor P53, DNA damage, xanthine oxidase, histological changes, oxidative stress and nitric oxide in rats: protective effect of ginseng," Food and Chemical Toxicology., vol. 78, pp. 17-25, 2015.

[14] F. Taghizadeh, S. J. Hosseinimehr, M. Zargari, A. Karimpour Malekshah, and F. B. Talebpour Amiri, "Gliclazide attenuates cisplatin-induced nephrotoxicity through inhibiting NF- $\kappa \mathrm{B}$ and caspase-3 activity," IUBMB Life, vol. 72, no. 9, pp. 20242033, 2020.

[15] P. D. Sanchez-Gonzalez, F. J. Lopez-Hernandez, F. Perez-Barriocanal, A. I. Morales, and J. M. Lopez-Novoa, "Quercetin reduces cisplatin nephrotoxicity in rats without compromising its anti-tumour activity," Nephrology Dialysis Transplantation., vol. 26, no. 11, pp. 3484-3495, 2011.

[16] B. H. Ali and M. S. Al Moundhri, "Agents ameliorating or augmenting the nephrotoxicity of cisplatin and other platinum compounds: a review of some recent research," Food and chemical toxicology., vol. 44, no. 8, pp. 1173-1183, 2006.

[17] Z. Wang, W. Sun, X. Sun, Y. Wang, and M. Zhou, "Kaempferol ameliorates cisplatin induced nephrotoxicity by modulating oxidative stress, inflammation and apoptosis via ERK and NF- $\kappa$ B pathways," AMB Express, vol. 10, no. 1, pp. 1-11, 2020.

[18] A. G. Casanova, M. T. Hernández-Sánchez, F. J. López-Hernández et al., "Systematic review and meta-analysis of the efficacy of clinically tested protectants of cisplatin nephrotoxicity," European journal of clinical pharmacology., vol. 76, no. 1, pp. 23-33, 2020.

[19] J. Sun, Y.-F. Chu, X. Wu, and R. H. Liu, "Antioxidant and antiproliferative activities of common fruits," Journal of agricultural and food chemistry., vol. 50, no. 25, pp. 7449-7454, 2002.

[20] K. Wolfe, X. Wu, and R. H. Liu, "Antioxidant activity of apple peels," Journal of agricultural and food chemistry., vol. 51, no. 3, pp. 609-614, 2003.

[21] C. Manach, G. Williamson, C. Morand, A. Scalbert, and C. Rémésy, "Bioavailability and bioefficacy of polyphenols in humans. I. Review of 97 bioavailability studies," The American journal of clinical nutrition., vol. 81, no. 1, pp. 230S-242S, 2005.

[22] G. V. Haute, E. Caberlon, E. Squizani et al., "Gallic acid reduces the effect of LPS on apoptosis and inhibits the formation of neutrophil extracellular traps," Toxicology In Vitro., vol. 30, no. 1, pp. 309-317, 2015.

[23] H. Asci, O. Ozmen, H. Y. Ellidag, B. Aydin, E. Bas, and N. Yilmaz, "The impact of gallic acid on the methotrexateinduced kidney damage in rats," journal of food and drug analysis, vol. 25, no. 4, pp. 890-897, 2017.

[24] S. Choubey, S. Goyal, L. R. Varughese, V. Kumar, A. K. Sharma, and V. Beniwal, "Probing gallic acid for its broad spectrum applications," Mini reviews in medicinal chemistry., vol. 18, no. 15, pp. 1283-1293, 2018.

[25] V. V. Padma, P. Sowmya, T. A. Felix, R. Baskaran, and P. Poornima, "Protective effect of gallic acid against lindane induced toxicity in experimental rats," Food and Chemical Toxicology., vol. 49, no. 4, pp. 991-998, 2011.

[26] A. Nouri, F. Heibati, and E. Heidarian, "Gallic acid exerts antiinflammatory, anti-oxidative stress, and nephroprotective effects against paraquat-induced renal injury in male rats," Naunyn-Schmiedeberg's Archives of Pharmacology., vol. 394, no. 1, pp. 1-9, 2021.

[27] R. P. Amin, A. E. Vickers, F. Sistare et al., "Identification of putative gene based markers of renal toxicity," Environmental health perspectives., vol. 112, no. 4, pp. 465-479, 2004.

[28] E.-J. Wang, R. D. Snyder, M. R. Fielden, R. J. Smith, and Y.$\mathrm{Z}$. Gu, "Validation of putative genomic biomarkers of nephrotoxicity in rats," Toxicology, vol. 246, no. 2-3, pp. 91-100, 2008.

[29] S. B. Bratton and G. M. Cohen, "Apoptotic death sensor: an organelle's alter ego?," Trends in Pharmacological Sciences., vol. 22, no. 6, pp. 306-315, 2001.

[30] W. Arjumand, A. Seth, and S. Sultana, "Rutin attenuates cisplatin induced renal inflammation and apoptosis by reducing NF $\kappa$ B, TNF- $\alpha$ and caspase- 3 expression in Wistar rats," Food and chemical toxicology., vol. 49, no. 9, pp. 20132021, 2011. 
[31] G. P. Kaushal, V. Kaushal, X. Hong, and S. V. Shah, "Role and regulation of activation of caspases in cisplatin-induced injury to renal tubular epithelial cells," Kidney international., vol. 60, no. 5, pp. 1726-1736, 2001.

[32] J. A. Buege and S. D. Aust, Microsomal lipid peroxidation, Methods in enzymology, Elsevier, 1978.

[33] G. L. Ellman, "Tissue sulfhydryl groups," Archives of biochemistry and biophysics., vol. 82, no. 1, pp. 70-77, 1959.

[34] R. Naserzadeh, L. Jafaripour, Z. Eslamifar, E. Alizamani, N. Nouryazdan, and $\mathrm{H}$. Ahmadvand, "The effect of receiving L-glutamine on the reduction of renal tissue damages and renal function recovery following gentamicin-induced nephrotoxicity in rats," Journal of Babol University of Medical Sciences, vol. 23, no. 1, pp. 267-274, 2021.

[35] J. Rotruck, A. Pope, H. E. Ganther, A. Swanson, D. G. Hafeman, and W. Hoekstra, "Selenium: biochemical role as a component of glutathione peroxidase," Science, vol. 179, no. 4073, pp. 588-590, 1973.

[36] M. Masayasu and Y. Hiroshi, "A simplified assay method of superoxide dismutase activity for clinical use," Clinica Chimica Acta., vol. 92, no. 3, pp. 337-342, 1979.

[37] H. Ahmadvand, E. Babaeenezhad, M. Nasri, L. Jafaripour, and K. R. Mohammadrezaei, "Glutathione ameliorates liver markers, oxidative stress and inflammatory indices in rats with renal ischemia reperfusion injury," Journal of Renal Injury Prevention, vol. 8, no. 2, pp. 91-97, 2019.

[38] Z. Eslamifar and S. Sabbagh, "A histopathological study of cisplatin-induced acute vascular injuries in vital organs and protective effect of Achillea millefolium," Journal of Pharmaceutical Research International., pp. 56-69, 2020.

[39] X.-j. Mi, J.-g. Hou, Z. Wang et al., "The protective effects of maltol on cisplatin-induced nephrotoxicity through the AMPK-mediated PI3K/Akt and p53 signaling pathways," Scientific Reports, vol. 8, no. 1, p. 15922, 2018.

[40] M. Piccart, H. Lamb, and J. B. Vermorken, "Current and future potential roles of the platinum drugs in the treatment of ovarian cancer," Annals of Oncology., vol. 12, no. 9, pp. 1195-1203, 2001.

[41] B. Ortega-Domínguez, O. E. Aparicio-Trejo, F. E. GarcíaArroyo et al., "Curcumin prevents cisplatin-induced renal alterations in mitochondrial bioenergetics and dynamic," Food and Chemical Toxicology., vol. 107, no. Part A, pp. 373-385, 2017.

[42] C. E. Bernal-Barquero, G. J. Vazquez-Zapien, and M. M. MataMiranda, "Revision de las alteraciones en la expresion genica y vias apoptoticas provocadas en la nefrotoxicidad inducida por cisplatino," Nefrología (English Edition)., vol. 39, no. 4, pp. 362-371, 2019.

[43] N. R. Ridzuan, N. A. Rashid, F. Othman, S. B. Budin, F. Hussan, and S. L. Teoh, "Protective role of natural products in cisplatin-induced nephrotoxicity," Mini reviews in medicinal chemistry., vol. 19, no. 14, pp. 1134-1143, 2019.

[44] P. Kalra, R. Karwasra, Y. K. Gupta, S. B. Ray, and S. Singh, "Terminalia chebulasupplementation attenuates cisplatininduced nephrotoxicity in Wistar rats through modulation of apoptotic pathway," Natural product research., vol. 33, no. 11, pp. 1641-1645, 2019.

[45] R. Domitrović, O. Cvijanović, E. P. Pugel, G. B. Zagorac, H. Mahmutefendić, and M. Škoda, "Luteolin ameliorates cisplatin-induced nephrotoxicity in mice through inhibition of platinum accumulation, inflammation and apoptosis in the kidney," Toxicology, vol. 310, pp. 115-123, 2013.
[46] Z. H. A. O. L. I. GAO, G. U. A. N. G. Y. I. LIU, Z. H. A. O. HU et al., "Grape seed proanthocyanidin extract protects from cisplatin-induced nephrotoxicity by inhibiting endoplasmic reticulum stress-induced apoptosis," Molecular medicine reports., vol. 9, no. 3, pp. 801-807, 2014.

[47] R. S. Barberino, V. G. Menezes, A. E. A. S. Ribeiro et al., "Melatonin protects against cisplatin-induced ovarian damage in mice via the MT1 receptor and antioxidant activity," Biology of Reproduction, vol. 96, no. 6, pp. 1244-1255, 2017.

[48] M. Saif-Elnasr, N. Abdel-Aziz, and A. I. El-Batal, “Ameliorative effect of selenium nanoparticles and fish oil on cisplatin and gamma irradiation-induced nephrotoxicity in male albino rats," Drug and chemical toxicology., vol. 42, no. 1, pp. 94-103, 2019.

[49] L. N. Al-Eitan, K. H. Alzoubi, L. I. Al-Smadi, and Khabour OF, "Vitamin E protects against cisplatin-induced genotoxicity in human lymphocytes," Toxicology In Vitro., vol. 62, p. $104672,2020$.

[50] M. A. Adefisayo, W. J. Adeyemi, and Q. K. Alabi, “Combined but not single administration of vitamin $\mathrm{C}$ and l-carnitine ameliorates cisplatin-induced gastric mucosa damage in male rats," Canadian journal of physiology and pharmacology., vol. 96, no. 8, pp. 830-838, 2018.

[51] N. Hooshyar, M. Sedighi, M. Hooshmand et al., "Mechanistic impact of medicinal plants affecting cisplatin-induced nephrotoxicity; an overview," Immunopathologia Persa, vol. 5, no. 1, 2019.

[52] R. S. Said, E. M. Mantawy, and E. El-Demerdash, "Mechanistic perspective of protective effects of resveratrol against cisplatininduced ovarian injury in rats: emphasis on anti-inflammatory and anti-apoptotic effects," Naunyn-Schmiedeberg's archives of pharmacology., vol. 392, no. 10, pp. 1225-1238, 2019.

[53] S. A. Habib, R. S. Abdelrahman, M. Abdel Rahim, and G. M. Suddek, "Anti-apoptotic effect of vinpocetine on cisplatininduced hepatotoxicity in mice: the role of annexin- $\mathrm{V}$, caspase-3, and Bax," Journal of Biochemical and Molecular Toxicology., vol. 34, no. 10, p. e22555, 2020.

[54] R. S. Abdelrahman, "Sitagliptin exerts anti-apoptotic effect in nephrotoxicity induced by cisplatin in rats," Naunyn-Schmiedeberg's archives of pharmacology., vol. 390, no. 7, pp. 721731, 2017.

[55] E. Bami, O. B. Ozakpınar, Z. N. Ozdemir-Kumral et al., "Protective effect of ferulic acid on cisplatin induced nephrotoxicity in rats," Environmental Toxicology and Pharmacology, vol. 54, pp. 105-111, 2017.

[56] S. A. Habib, G. M. Suddek, M. A. Rahim, and R. S. Abdelrahman, "The protective effect of protocatechuic acid on hepatotoxicity induced by cisplatin in mice," Life Sciences., vol. 277, p. 119485, 2021.

[57] H.-K. Shin, T.-W. Kim, Y.-J. Kim, S.-R. Park, C.-S. Seo, and H. Ha, "Protective effects of dendrobium nobile against cisplatin nephrotoxicity both in-vitro and in-vivo," Iranian journal of pharmaceutical research: IJPR., vol. 16, Supplement, p. 197, 2017.

[58] Y. Yilmaz and R. T. Toledo, "Major flavonoids in grape seeds and skins: antioxidant capacity of catechin, epicatechin, and gallic acid," Journal of agricultural and food chemistry., vol. 52, no. 2, pp. 255-260, 2004.

[59] N. Kahkeshani, F. Farzaei, M. Fotouhi, S. S. Alavi, R. Bahramsoltani, and R. Naseri, "Pharmacological effects of gallic acid in health and diseases: a mechanistic review," Iranian journal of basic medical sciences., vol. 22, no. 3, pp. 225237, 2019. 
[60] B. Badhani, N. Sharma, and R. Kakkar, "Gallic acid: a versatile antioxidant with promising therapeutic and industrial applications," Rsc Advances., vol. 5, no. 35, pp. 27540-27557, 2015.

[61] J. Gao, J. Hu, D. Hu, and X. Yang, "A role of gallic acid in oxidative damage diseases: a comprehensive review," Natural Product Communications, vol. 14, no. 8, article 1934578X1987417, 2019.

[62] O. Karimi-Khouzani, E. Heidarian, and S. A. Amini, “Antiinflammatory and ameliorative effects of gallic acid on fluoxetine- induced oxidative stress and liver damage in rats," Pharmacological reports., vol. 69, no. 4, pp. 830-835, 2017.

[63] B. Gholamine, G. Houshmand, A. Hosseinzadeh, M. Kalantar, S. Mehrzadi, and M. Goudarzi, "Gallic acid ameliorates sodium arsenite-induced renal and hepatic toxicity in rats," Drug and chemical toxicology., vol. 44, no. 4, pp. 341-352, 2021.

[64] T. T. G. Nieskens, J. G. P. Peters, D. Dabaghie et al., "Expression of organic anion transporter 1 or 3 in human kidney proximal tubule cells reduces cisplatin sensitivity," Drug Metabolism and Disposition., vol. 46, no. 5, pp. 592-599, 2018.

[65] G. Sindhu, E. Nishanthi, and R. Sharmila, "Nephroprotective effect of vanillic acid against cisplatin induced nephrotoxicity in Wistar rats: a biochemical and molecular study," Environmental Toxicology and Pharmacology, vol. 39, no. 1, pp. 392404, 2015.

[66] T. Neamatallah, N. El-Shitany, A. Abbas et al., "Nano ellagic acid counteracts cisplatin-induced upregulation in OAT1 and OAT3: a possible nephroprotection mechanism," Molecules, vol. 25, no. 13, p. 3031, 2020.

[67] H. Allameh, I. Fatemi, A. R. Malayeri, A. Nesari, S. Mehrzadi, and M. Goudarzi, "Pretreatment with berberine protects against cisplatin-induced renal injury in male Wistar rats," Naunyn-Schmiedeberg's archives of pharmacology., vol. 393, no. 10, pp. 1825-1833, 2020.

[68] I. K. Mohan, M. Khan, J. C. Shobha et al., "Protection against cisplatin-induced nephrotoxicity by spirulina in rats," Cancer Chemotherapy and Pharmacology, vol. 58, no. 6, pp. 802808, 2006.

[69] J. C. Ruiz-Ruiz, A. J. Matus-Basto, P. Acereto-Escoffié, and M. R. Segura-Campos, "Antioxidant and anti-inflammatory activities of phenolic compounds isolated fromMelipona beecheiihoney," Food and Agricultural Immunology., vol. 28, no. 6, pp. 1424-1437, 2017.

[70] J. Bai, Y. Zhang, C. Tang et al., "Gallic acid: pharmacological activities and molecular mechanisms involved in inflammation-related diseases," Biomedicine \& Pharmacotherapy., vol. 133, p. 110985, 2021.

[71] O. R. Kunduzova, G. Escourrou, M.-H. Seguelas et al., "Prevention of apoptotic and necrotic cell death, caspase-3 activation, and renal dysfunction by melatonin after ischemia/reperfusion," The FASEB journal., vol. 17, no. 8, pp. 1-17, 2003.

[72] M. Khan, S. Varadharaj, J. C. Shobha et al., "C-Phycocyanin ameliorates doxorubicin-induced oxidative stress and apoptosis in adult rat cardiomyocytes," Journal of cardiovascular pharmacology., vol. 47, no. 1, pp. 9-20, 2006. 